\title{
A fatal case with multiple liver and splenic abscesses due to Streptococcus anginosus bacteremia in a patient with diabetic ketoacidosis
}

\author{
Chien-Wen Yang, Wasique Mirza, Mladen Jecmenica, Gagandeep Dhillon, Mahesh Cheryala, Ripudaman Munjal and Qi Shi* \\ The Wright Center for Graduate Medical Education, Department of Medicine, USA
}

\begin{abstract}
Long standing hyperglycemia can be a risk factor for abscess formation, and abscess can further increase serum glucose levels. This is a fatal case of multiple liver and splenic abscesses from Streptococcus anginosus bacteremia in a patient presenting with diabetic ketoacidosis and elevated liver enzymes.
\end{abstract}

\section{Introduction}

A common etiology of diabetic ketoacidosis is severe infection [1], and hyperglycemia has been reported to be a possible risk factor for developing a liver abscess [2]. Streptococcus anginosus is a subgroup of streptococcus viridians, which commonly exists in normal oral and gastrointestinal flora. Streptococcus anginosus differs from other bacterial subgroups as Streptococcus anginosus may cause abscess formation [3]. A review of the literature presents few case reports regarding Streptococcus anginosus bacteremia and its association with liver abscess $[4,5]$. This is a fatal case presentation of a patient with diabetic ketoacidosis who developed multiple liver and splenic abscesses from Streptococcus anginosus bacteremia.

\section{Case presentation}

A 63-year-old male presented to the emergency room with generalized weakness, polydipsia and polyuria for one week. $\mathrm{He}$ denied fever, chills, cough, sore throat, chest pain, nausea, vomiting, diarrhea, abdominal pain, or contact with individuals who were ill. The patient had a history of diabetes mellitus and complete heart block s/p pacemaker insertion in 2015. He had stopped taking metformin and glipizide for several weeks due to insurance issues. On admission, the patient was afebrile, with a temperature of 97.4 degrees Fahrenheit, heart rate of 96 beats per minute and respiratory rate of 18 breaths per minute. The patient's blood pressure was $107 / 57 \mathrm{mmHg}$ and oxygen saturation was $96 \%$ on ambient air. The physical exam was negative except for dry oral mucous membranes and the patient having an acutely ill appearance. Lab results showed: WBC $13.37 \mathrm{~K} / \mathrm{uL}, \mathrm{Hgb}$ $14.7 \mathrm{~g} / \mathrm{dl}$, Platelets $183 \mathrm{~K} / \mathrm{uL}$, BUN $69 \mathrm{mg} / \mathrm{dL}$, Creatinine $1.8 \mathrm{mg} /$ dL (baseline 1.0), AST $125 \mathrm{U} / \mathrm{L}$, ALT $139 \mathrm{U} / \mathrm{L}$, ALP $264 \mathrm{U} / \mathrm{L}$, total bilirubin $0.8 \mathrm{mg} / \mathrm{dL}$, anion gap 28, HbA1c $14.5 \mathrm{mg} / \mathrm{dL}$, serum glucose $462 \mathrm{mg} / \mathrm{dL}, \mathrm{CO}_{2} 13 \mathrm{mmol} / \mathrm{L}$ and positive urine ketones. The patient was admitted and started on the standard treatment for diabetic ketoacidosis. On the second day of hospitalization, an abdominal ultrasound showed heterogeneous hypoechoic lesions in the liver and an abdominal computerized tomography scan was ordered. On the third day of hospitalization, the patient developed a fever of 102 degrees Fahrenheit and a HR of 180 beats per minute. The systolic blood pressure dropped to $80 \mathrm{~mm} \mathrm{Hg}$. The patient was started on intravenous Vancomycin, Cefepime and Flagyl. Blood culture results were positive for Streptococcus anginosus. An abdominal computerized tomography scan with intravenous contrast showed multiple peripherally enhanced low density lesions throughout the liver with the largest measuring 9.6 x $7.5 \mathrm{~cm}$, and an enlarged spleen with an 8.7 x 3.7 x $3.9 \mathrm{~cm}$ low density multi-loculated fluid collection (Figure 1). Core needle biopsy showed polytypic plasma cell proliferation with micro-abscesses compatible with the clinical suspicion of liver abscesses. Additional evaluation for malignancy included tumor markers, serum/urine protein electrophoresis, and peripheral blood flow cytometry. Results of the tests were negative. General Surgery was consulted but no surgery was

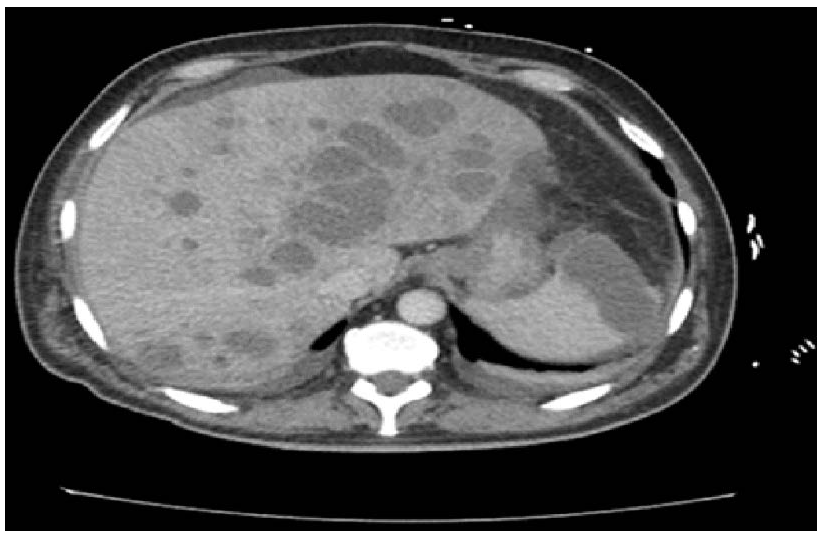

Figure 1. Multiple peripheral enhanced low density lesions throughout liver and an enlarged spleen with a low density multiloculated fluid collection showed on abdominal CAT scan with IV contrast.

Correspondence to: Qi Shi, The Wright Center for Graduate Medical Education, Department of Medicine, 501 Madison Ave, Scranton, PA, USA, Tel: +1 570468-4867; Fax: +1 570-343-4800; E-mail: shiq@thewrightcenter.org

Key words: Streptococcus anginosus, liver abscess, splenic abscess, bacteremia, diabetic ketoacidosis

Received: March 05, 2017; Accepted: March 20, 2017; Published: March 23, 2017 
planned in view of the extensive nature of the lesions and poor clinical condition. A hepatic drainage tube was placed and purulent exudative fluid was drained. Cultures performed on the drainage fluid showed gram positive cocci, which failed to grow on subculture. The patient died due to refractory septic shock on day 22 of hospitalization despite aggressive treatment with antibiotics.

\section{Discussion}

Severe infection is one of the most common etiologies of diabetic ketoacidosis [1]. The possible mechanisms may include increasing glycogenolysis, stress induced physiological steroid production, peripheral insulin resistance etc [6]. In our reported case, the HbAlc on admission was 14.5 , which implies long standing hyperglycemia before abscess formation. The development of multiple abscesses further increased the serum glucose levels, adding to the risk of metabolic acidosis.

The patient did not present with any fever, or abdominal symptoms on admission. Any patient presenting with diabetic ketoacidosis and abnormal liver functions may warrant prompt abdominal imaging or empiric antibiotics treatment.

Streptococcus anginosus has the unique characteristic of producing abscesses, which distinguishes it from other bacteria in the Streptococcus Viridians subgroups [3]. Common sites of abscess formation are brain, lung, liver and spleen. Underlying conditions associated with abscess formation include dental infection, malignancy and gastrointestinal diseases, which were not identified in our patient [7-9]. Several cases are reported worldwide with coexisting brain-liver, brain-lung and brain-spleen abscess with or without bacteremia [5]. This case is unique as the patient developed multiple liver and spleen abscesses with Streptococcus anginosus bacteremia.

The pathology report demonstrated plasma cell proliferation with micro abscesses. Plasma cells play an important role in producing antibodies in the in adaptive immune response. The association between plasma cell proliferation and liver abscess is unknown. Also, in our case, further evaluation for plasma cell disorders, including tumor markers, serum/urine protein electrophoresis, and peripheral blood flow cytometry demonstrated negative results [10].

A dilemma was encountered during treatment. Per the current treatment guidelines, image-guided percutaneous drainage or surgical drainage were recommended due to poor penetration of antibiotics [11]. In reported cases, drainage of the abscess with intravenous antibiotics (Penicillin or Cephalosporin) had a high rate of cure [5]. However, in our patient, there were multiple abscesses throughout the liver, which made either percutaneous drainage (failure to drain all the abscesses found) or surgical drainage (possible need to sacrifice the whole liver) extremely challenging. Despite intravenous antibiotic administration and two percutaneous abscess drainages, the patient died of profound septic shock.

\section{Conclusion}

This case suggests that long standing hyperglycemia can be a risk factor for liver and splenic abscess formation associated with Streptococcus anginosus bacteremia. This was further complicated by the development of diabetic ketoacidosis and this combination was life-threatening. The authors recommend that any patient presenting with diabetic ketoacidosis and an acute elevation of liver enzymes be evaluated by prompt abdominal imaging studies with initiation of antibiotic therapy to decrease the morbidity and mortality in this population. There is a further need to follow similar case reports, which may enlighten provide a better understanding of etiology and treatment options.

\section{References}

1. Kakusa, M, Kamanga B2, Ngalamika O3, Nyirenda S (2016) Comatose and noncomatose adult diabetic ketoacidosis patients at the University Teaching Hospital, Zambia: Clinical profiles, risk factors, and mortality outcomes. Indian J Endocrinol Metab 20: 199-205. [Crossref]

2. Chu KM, Fan ST, Lai EC, Lo CM, Wong J (1996) Pyogenic liver abscess. An audit of experience over the past decade. Arch Surg 131: 148-152. [Crossref]

3. Gossling J (1988) Occurrence and pathogenicity of the Streptococcus milleri group. Rev Infect Dis 10: 257-285. [Crossref]

4. Nisbet M, Thomas M (2005) Liver abscess associated with persistent Streptococcus anginosus bacteremia. Clin Infect Dis 41: 352-3, 403-5. [Crossref]

5. Giuliano S, Rubini G, Conte A, Goldoni P, Falcone M, et al. (2012) Streptococcus anginosus group disseminated infection: case report and review of literature. Infez Med 20: 145-54. [Crossref]

6. Buczkowska EO (2002) [Alterations of blood glucose homeostasis during septic or injury stress--hyperglycemia]. Wiad Lek 55: 731-744. [Crossref]

7. Iwashita H, Matsui N, Tsukamoto S, Funakoshi S, Yamaguchi M, et al. (2015) [A case of liver abscess due to Streptococcus anginosus infection secondary to a dental extraction]. Nihon Shokakibyo Gakkai Zasshi 112: 1525-32. [Crossref]

8. Livingston LV, Perez-Colon E (2014) Streptococcus intermedius Bacteremia and Liver Abscess following a Routine Dental Cleaning. Case Rep Infect Dis. [Crossref]

9. Zhou Z, Wuppalapati S, Scott N (2015) Snapshot in surgery: brain abscess as a complication of a recurrent sigmoid diverticular abscess. Clin Case Rep 3: 506-7. [Crossref]

10. Roth K, Oehme L, Zehentmeier S, Zhang Y, Niesner R, et al. (2014) Tracking plasma cell differentiation and survival. Cytometry $A$ 85: 15-24. [Crossref]

11. Singh S, Chaudhary P, Saxena N, Khandelwal S, Poddar DD et al. (2013) Treatment of liver abscess: prospective randomized comparison of catheter drainage and needle aspiration. Ann Gastroenterol 26: 332-339. [Crossref]

Copyright: (C2017 Yang CW. This is an open-access article distributed under the terms of the Creative Commons Attribution License, which permits unrestricted use, distribution, and reproduction in any medium, provided the original author and source are credited. 